\title{
Dissociating the Human Language Pathways with High Angular Resolution Diffusion Fiber Tractography
}

\author{
Stephen Frey, ${ }^{1}$ Jennifer S. W. Campbell, ${ }^{2}$ G. Bruce Pike, ${ }^{2}$ and Michael Petrides ${ }^{1}$ \\ ${ }^{1}$ Cognitive Neuroscience Unit and ${ }^{2}$ McConnell Brain Imaging Centre, Montreal Neurological Institute, McGill University, Montreal, Quebec, Canada H3A 2B4
}

The anatomical connectivity of ventrolateral frontal areas 44 and 45 , which in the human brain constitute Broca's region, has been revisited on the basis of experimental anatomical tracer evidence in the nonhuman primate that the homologues of areas 44 and 45 have distinct bidirectional corticocortical connections. Here we show, using high angular resolution diffusion imaging in the living human brain, a dissociation between the specific projections from the pars opercularis (area 44) and the pars triangularis (area 45) in the ventrolateral frontal lobe. As in the macaque monkey, area 44 has distinct connections with the rostral inferior parietal lobule via the third branch of the superior longitudinal fasciculus. In contrast, area 45 connects with the superior temporal gyrus, anterior to Heschl's gyrus, via the extreme capsule fiber system. These results highlight the differences in connectivity between areas 44 and 45 which had previously been thought to be uniformly connected with the posterior temporal region via the arcuate fasciculus. We also provide evidence in the human brain that the arcuate fasciculus, as in the macaque monkey brain, connects the posterior superior temporal region with dorsolateral frontal areas 8 and rostral 6 that lie above areas 44 and 45 . Thus, monkey and human evidence suggests that the connections of areas 44 and 45 are much more differentiated than had previously been thought and provide the basis for studies searching for their differential contribution in function.

Key words: Broca's; Wernicke's; arcuate fasciculus; language; frontal; auditory

\section{Introduction}

The ventrolateral frontal region in the left hemisphere of the human brain, Broca's region, is critically involved in various aspects of expressive language processing (Grodzinsky and Amunts, 2006). This frontal language zone has traditionally been thought to be linked with the temporal language region via the arcuate fasciculus (Geschwind, 1970). Recently, several groups have investigated the connections between the posterior temporal language zone and the anterior frontal language zone using diffusion tensor imaging (DTI), a noninvasive method of investigating white matter fiber tracts in vivo (Catani et al., 2005; Croxson et al., 2005; Friederici et al., 2006). The above investigations focused on the temporo-frontal arching pathway that is thought to link Wernicke's with Broca's region, namely the arcuate fasciculus (Geschwind, 1970). Some diffusion studies suggested that there may also be a "ventral pathway" connecting language areas of the frontal and temporal lobe via the uncinate fasciculus, the external capsule, or the extreme capsule (Croxson et al., 2005; Parker et al., 2005; Friederici et al., 2006; Powell et al., 2006; Anwander et al., 2007; Rilling et al., 2008).

Broca's region includes two distinct architectonic areas: area 44 , a dysgranular cortical area occupying the posterior part of

Received May 27, 2008; revised Sept. 16, 2008; accepted Sept. 21, 2008.

This work was supported by the Canadian Institutes of Health Research (MOP-14620) and the Natural Sciences and Engineering Research Council of Canada (RGPIN 170426-07).

Correspondence should be addressed to Dr. Stephen Frey, Montreal Neurological Institute, McGill University, 3801 University Street, Montreal, Quebec, Canada H3A 2B4. E-mail: stephen@bic.mni.mcgill.ca. DOI:10.1523/JNEUROSCI.2388-08.2008

Copyright $\odot 2008$ Society for Neuroscience ～0270-6474/08/2811435-10\$15.00/0
Broca's region, and area 45, a granular cortical area occupying the anterior part of this region (Petrides and Pandya, 1994; Amunts et al., 1999). The homologues of these two architectonic areas in the macaque monkey have been established by comparative architectonic studies (Petrides and Pandya, 1994, 2002a; Petrides et al., 2005). Experimental anatomical tracer studies in the macaque monkey demonstrated that the homologues of areas 44 and 45 have quite distinct bidirectional corticocortical connections. Petrides and Pandya (1984) first showed that there is a major branch of the superior longitudinal fasciculus originating from the rostral part of the inferior parietal lobule (SLF III) which targets the ventral premotor zone including the depth of the ventral branch of the arcuate sulcus where the homolog of area 44 has now been shown to be located (Petrides et al., 2005). These connections are bidirectional (Petrides and Pandya, 2002b; S. Frey, M. Mackey, and M. Petrides, unpublished studies). In contrast, the more anterior ventrolateral prefrontal region that includes the homolog of area 45 is linked with the middle and posterior section of the superior temporal gyrus via a distinct pathway that runs through the extreme capsule (Petrides and Pandya, 1988). In the macaque monkey, the arcuate fasciculus curves around the posterior end of the Sylvian fissure and is linked with caudolateral frontal areas 8 and rostral area 6 that lie just above area 44 (Petrides and Pandya, 1988, 2002b; Romanski et al., 1999a) (Fig. 1a). We reinvestigate the pathways that connect Broca's zone in the ventrolateral frontal lobe with posterior cortical regions in the human brain using a high angular resolution diffusion imaging technique, q-ball imaging (Tuch et al., 2003), to examine whether the posterior (area 44) and anterior (area 45) parts of Broca's zone 
have distinct connections via two separate pathways as the macaque monkey experimental anatomical data would predict.

\section{Materials and Methods}

Data acquisition. We used fiber tractography to establish the connectivity patterns between individual regions of interest (ROIs) that were drawn onto the $\mathrm{T} 1$ weighted anatomical magnetic resonance imaging scans of 12 healthy subjects ( 6 female and 6 male, mean age 29.8, range 23-39 years) using a Siemens 3 T Trio MR scanner and an 8-channel phased-array head coil. Diffusion encoding was achieved using a single-shot spin-echo echo-planar sequence with twice-refocused balanced gradients. There were 99 diffusion encoding directions with 2 $\mathrm{mm}$ isotropic voxel size, 63 slices, $b=3000$ $\mathrm{s} / \mathrm{m}^{2}\left(q=0.35 \mu \mathrm{m}^{-1}\right), \mathrm{TE}=121 \mathrm{~ms}, \mathrm{TR}=$ $11.1 \mathrm{~s}$, GRAPPA parallel reconstruction. A 1 $\mathrm{mm}$ isotropic resolution $\mathrm{T} 1$ weighted scan was also acquired ( $\mathrm{TR}=9.7 \mathrm{~ms}$, $\mathrm{TE}=4 \mathrm{~ms}, \alpha=12$ degrees). The two datasets were registered using a mutual information based algorithm (Maes et al., 1997) to remove image misregistration from distortion and motion. The diffusion orientation distribution function (ODF) was calculated using q-ball reconstruction (Tuch at al., 2003) and the ODF was calculated at 99 isotropically spaced directions generated using an electrostatic repulsion algorithm. The diffusion tensor was also calculated at each voxel to generate fractional anisotropy and mean diffusivity maps.

Regions of interest. Regions of interest (ROIs) were drawn on each subject's T1 weighted anatomical magnetic resonance imaging scan using $3 \mathrm{D}$ visualization software (MacDonald, 1996). A large ROI that encompassed Broca's area was painted within the ventrolateral frontal cortex, anterior to the precentral sulcus, and included the pars opercularis (area 44) and the pars triangularis (area 45) of the inferior frontal gyrus. It should be noted that we made no assumptions as to the exact location of areas 44 and 45 within Broca's region, and it is for this reason that this large ROI was used for our tractography. This region is easy to identify on anatomical MRI scans because it is delimited by the inferior branch of the precentral sulcus posteriorly, the inferior frontal sulcus superiorly and the horizontal ramus of the Sylvian fissure ventrally. The pars opercularis, which forms the posterior part of the ROI, is separated from the pars triangularis, which forms the anterior part of the ROI, by the ascending ramus of the Sylvian fissure. Electrical stimulation of the pars opercularis and the caudal part of the pars triangularis during brain surgery results in speech arrest (Penfield and Roberts, 1966; Rasmussen and Milner, 1975; Ojemann et al., 1989) (Fig. 1b).

An ROI was also drawn in the inferior parietal lobule encompassing the supramarginal gyrus (i.e., area 40 or PF), which lies ventral to the intraparietal sulcus of the human brain (Fig. 1b). In the macaque monkey, the rostral part of the inferior parietal lobule that encompasses areas $\mathrm{PF}$ and PFG projects to the ventral premotor region in- a

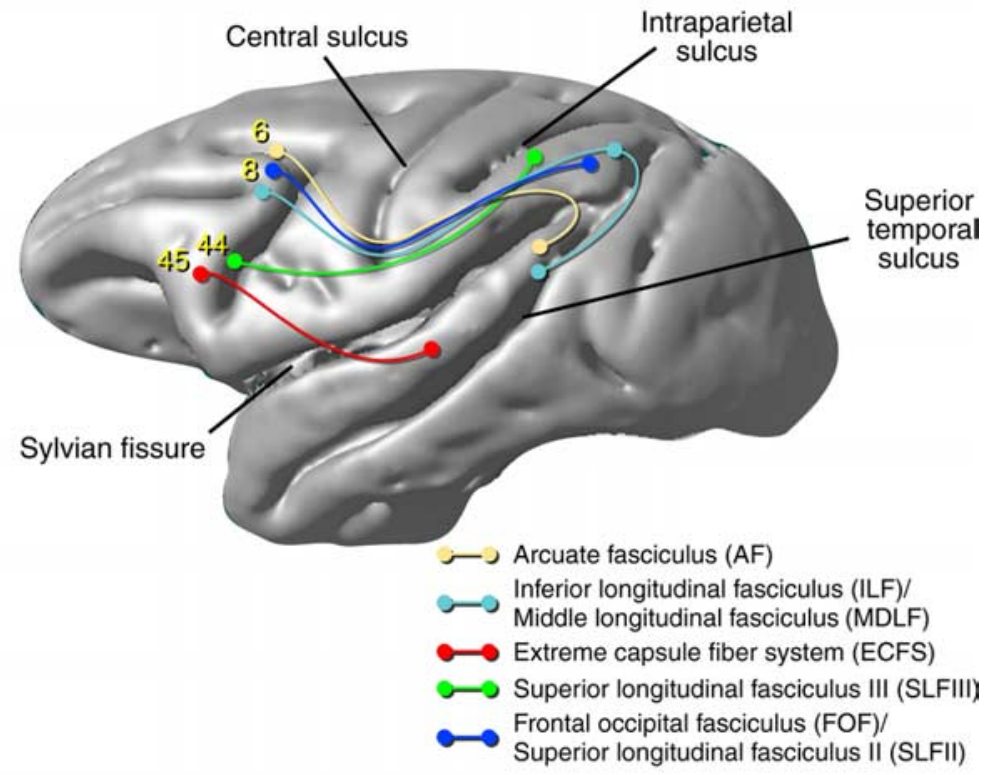

b

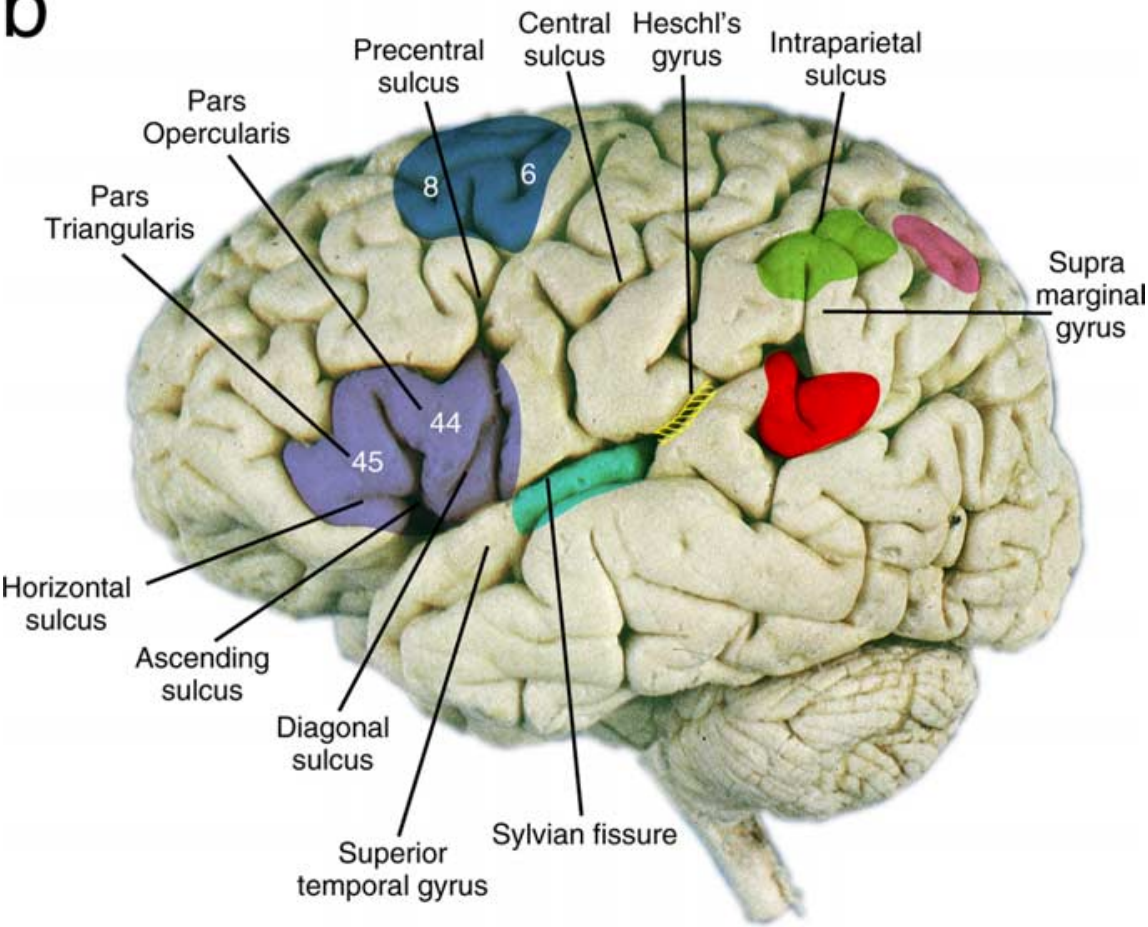

Regions of Interest (ROIs)
Broca's area
Superior temporal region (anterior to Heschl's gyrus)
Posterior superior temporal gyrus
Inferior parietal
Posterior parietal
Areas 8 \& 6

Figure 1. Schematic representations of the regions of interest (areas 44 and 45 ) in the human and monkey brains. $\boldsymbol{a}$, Experimental anatomical tract tracing in the monkey have demonstrated that the majority of the bidirectional connections of area 44 are made with regions in the anterior part of the inferior parietal lobule (the homolog of the supramarginal gyrus) via the third branch of the SLF III, whereas area 45 is connected to the superior temporal gyrus and sulcus via the ECFS. The AF, ECFS, FOF, SLFII and SLFIII, and MDLF, as well as the ILF are also presented in this diagram. $\boldsymbol{b}$, Illustration of the ROIs that were drawn on each subject's anatomical scan (color coded). 


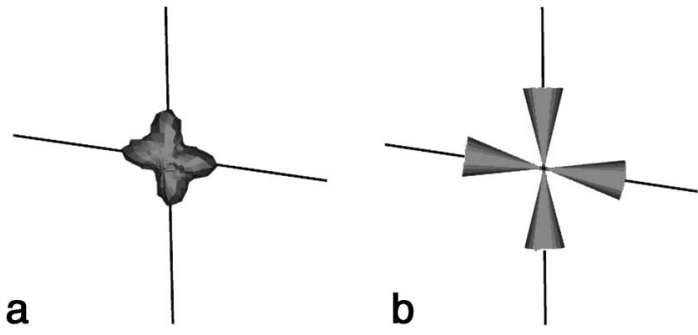

Figure 2. Tractography input. $\boldsymbol{a}$, The diffusion orientation distribution function is calculated using $Q B I$ reconstruction. The maxima are extracted, and streamlines are propagated in all directions within the solid angle dictated by the finite angular resolution of the acquisition. $\boldsymbol{b}$, The maximum and associated cone closest to the incoming streamline direction are chosen at each voxel. The voxel shown here indicates a two fiber crossing.

cluding the depth of the ventral branch of the arcuate sulcus (Petrides and Pandya, 1984) where the homolog of area 44 lies (Petrides et al., 2005). The rostral part of the inferior parietal lobule is involved in complex somatosensory responses of the face and arm (Hyvärinen and Shelepin, 1979; Leinonen and Nyman, 1979). The other ROI was placed within the mid-portion of the superior temporal gyrus and sulcus (i.e., area 22), anterior to Heschl's gyrus (Fig. 1b). In the macaque monkey, this region of the superior temporal gyrus is known to connect to the inferior frontal convexity (Petrides and Pandya, 1988, 2002b; Romanski et al., 1999a) where the homolog of area 45 is located (Petrides et al., 2005).

An additional ROI was defined in the left hemisphere to include the caudal parts of the superior and middle frontal gyri, anterior to the superior precentral sulcus. The aim here was to include the caudal dorsolateral frontal cortex (area 8 and rostral area 6), which are known to be connected with the most posterior part of the superior temporal gyrus via an arching fasciculus (Petrides and Pandya, 1988, 2006). An ROI was also defined on the most posterior aspect of the superior temporal gyrus, ventral to the end of the Sylvian fissure. Finally ROIs were defined in the posterior ventral aspect of the intraparietal sulcus located within the inferior parietal lobule (Fig. 1b). Note that all of the regions of interest included white matter immediately adjacent to the painted cortex to allow white matter fiber tracking.

Fiber reconstruction. We used a modified brute-force streamline tractography approach that uses the information on subvoxel crossing fibers obtained from the Q-Ball imaging reconstruction and which is an adaptation of our earlier approach (Campbell et al., 2006; Savadjiev et al., $2006,2008)$. The maxima of the diffusion ODF were considered to correspond to fiber directions. Because of the finite angular resolution of the acquisition, the precision on the location of the ODF maxima, and hence the inferred fiber directions, is not infinite. The tracking algorithm was therefore adapted to take into account this lack of precision (Fig. 2). The maxima were calculated as follows: the ODF was first smoothed by convolution with a Gaussian kernel to reduce extraneous maxima caused by noise. The full-width-half-max (FWHM) of the kernel was empirically chosen to be FWHM $=1.5 \times \sqrt{2 \pi / N}$, where $N$ is the number of directions sampled on the hemisphere. For $N=99$, this corresponded to $21.7^{\circ}$. The derivative of the ODF surface was then taken at each sampled direction and directions corresponding to maxima were retained. Because the true ODF maximum can lie anywhere in the solid angle $4 \pi / 2 \mathrm{~N}$ centered on the maximal direction, streamlines were propagated in all directions within this cone instead of only along the maximal vector (Fig. 2). This makes the tracking more robust to the finite angular resolution of the acquisition, hence minimizing false negatives caused by this confound. Streamlines were propagated using FACT integration (Mori et al., 1999). At each step, the ODF maximum closest to the incoming propagation direction was chosen. Ten thousand iterations per start voxel were used to fully sample the cones around all maxima and, on each iteration, the direction of propagation was chosen from within the cone surrounding the maximal direction chosen.

The brute force tractography approach (Conturo et al., 1999), which starts tract propagation in all voxels in a volume and retains those that pass through a region or regions of interest, can considerably reduce false-negative tractography results compared with starting in the tractdelineating ROIs only. For example, the tracking result obtained when starting in ROI A will be slightly different from that obtained when starting from ROI B, which will again be slightly different from that obtained if starting from the middle of the tract connecting them. All these differ in the location of the false-negative results, and combining them all will give a more accurate view of the fiber structure connecting $\mathrm{A}$ and $\mathrm{B}$. In the absence of knowledge of the location of the path connecting $A$ and $B$, starting every voxel in the brain is the most reliable approach. However, this approach is time consuming, especially when combined with iterative streamline propagation. The brute force approach was modified as follows. Each tracking experiment was performed to extract all tracts passing through both of the two ROIs in one subject. As a first step, streamlines were propagated starting in all voxels of both of the ROIs in question, and a binary connectivity profile of all voxels these streamlines passed through was saved. Next, tracking was initiated in every nonzero voxel from the result of step one and all tracts that passed through both ROIs were retained, producing a connectivity map of the connections passing through both ROIs (Fig. 3). The purpose of the first step was to generate a tract-specific and operator-independent starting region that was significantly smaller than the full brain, but much larger than the original start ROIs. This starting region encompasses most, but generally not all, of the path in question, and by restarting tractography in each voxel in it, a pathway comparable to that obtained using full brain brute force tractography can be obtained. The pairs of ROIs investigated were: (1) Broca's area and the rostral inferior parietal lobule, (2) Broca's area and the superior temporal gyrus and sulcus, (3) Broca's area and the posterior extent of the superior temporal gyrus, (4) area 8/6 and the posterior extent of the superior temporal gyrus, and (5) the posterior inferior parietal lobule and the posterior extent of the superior temporal gyrus (Fig. 1b).

As an additional measure to accelerate tracking, tracking was stopped if the midline of the brain was crossed, therefore only connections in the left hemisphere are considered. Tracking was stopped if the curvature of the tract exceeded $90^{\circ}$, if the fractional anisotropy was $<0.1$, or if the mean diffusivity was $>10^{-6} \mathrm{~mm}^{2} / \mathrm{ms}$ (to exclude voxels containing predominantly gray matter with little fiber directional coherence, or significant CSF).

Population connectivity maps. The T1 weighted anatomical scans were used to register the tracking results for all subjects' brains. The connectivity profiles were added to yield population connectivity maps for all the pathways (Fig. 4). For the connectivity maps of the Broca ROI with the two distinct ROIs that had been paired with it (rostral inferior parietal lobule and superior temporal gyrus and sulcus, respectively), a map of all voxels in the connectivity profile that lay within the Broca ROI was created. These maps were transformed to standard space to create a population segmentation map of all Broca ROI voxels connected to the rostral inferior parietal lobule ROI and all Broca ROI voxels connected to the superior temporal gyrus and sulcus. To create this population segmentation map, voxels that had more connections, in the population of 12 , to rostral inferior parietal lobule were labeled as such, and voxels with more connections to superior temporal gyrus and sulcus were labeled as such (Fig. 5).

\section{Results}

\section{Area 44 and 45 projections}

In 10 of 12 subjects, connections were seen between the Broca ROI and the ROI in the rostral inferior parietal lobule (namely the supramarginal gyrus). These connections were made with the most posterior part of the Broca ROI (i.e., the region that included the pars opercularis on which area 44 is known to lie) (Fig. 3 ). These projections followed the third branch of the superior longitudinal fasciculus (SLF III) that was originally demonstrated in experimental anatomical tracing studies using the autoradiographic method in the macaque monkey (Petrides and Pandya, 1984). Additionally, in 10 of 12 subjects, connections were observed between the Broca ROI and the ROI located within the superior temporal gyrus and sulcus. These connections were 


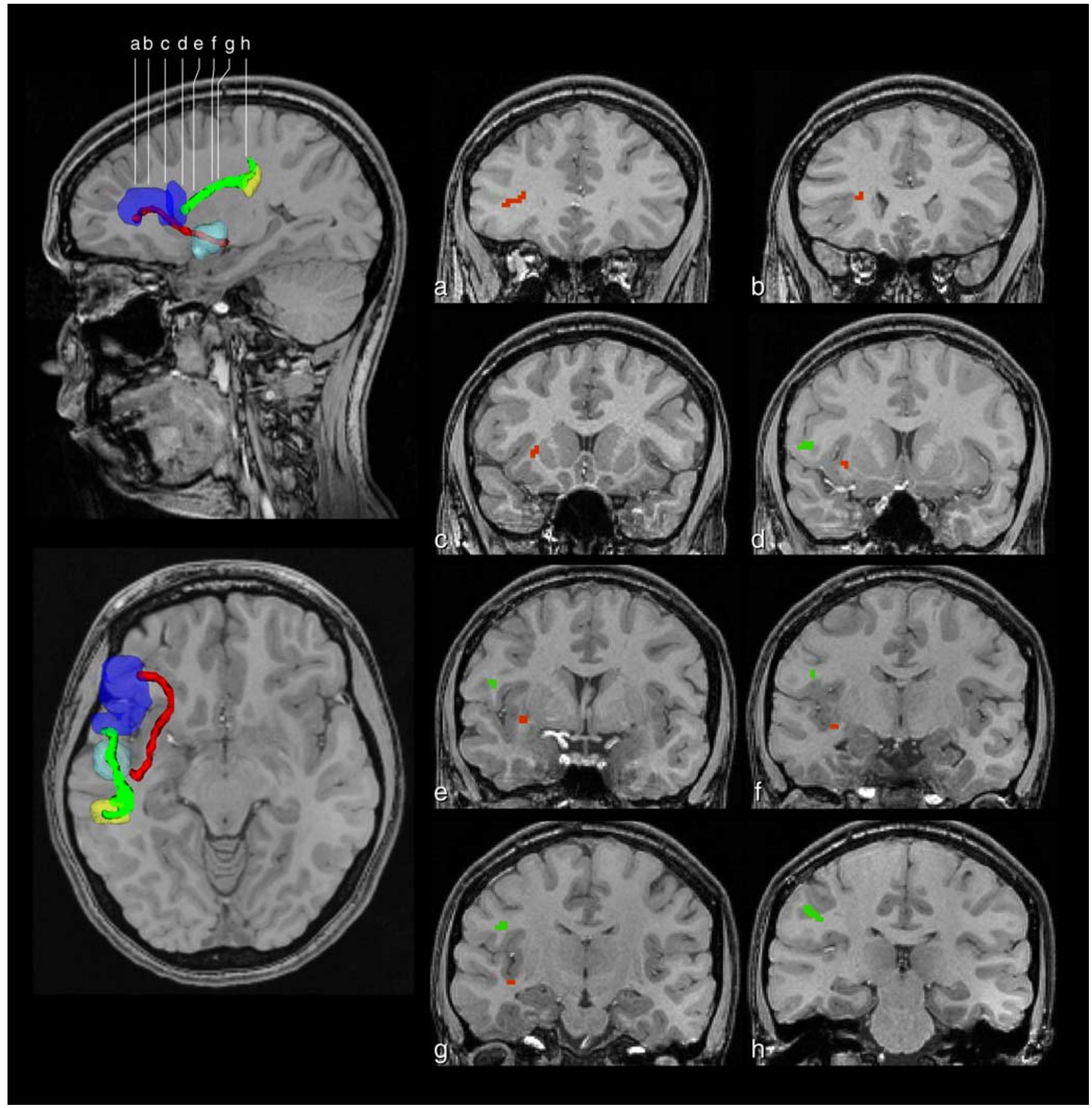

Figure 3. Tractography in the left hemisphere of a single subject for areas 44 and 45. The tractography revealed that the connections (shown in green) between the large Broca area R0I (purple) and the ROI in the rostral inferior parietal lobule within the supramarginal gyrus (yellow) began mainly in area 44. In contrast, the connections (shown in red) between the large Broca area R0l and the ROI in the superior temporal gyrus and sulcus originated mainly in area 45. The colored pathways are surfaces encompassing all nonzero voxels in the connectivity map for each connection. Although the tract data displayed here are projected on a single plane, investigation using our 3D tract display demonstrated that projections from area 44 likely travel along the SLF III to the inferior parietal cortex. In contrast, the projections of area 45 traveled through the ECFS into the superior temporal gyrus and sulcus, anterior to Heschl's gyrus. This is demonstrated in the coronal sections within a single subject $(\boldsymbol{a}-\boldsymbol{h})$.

made with the most anterior part of the Broca ROI (i.e., the region that included the pars triangularis on which area 45 lies (Fig. 3). These projections followed a course through the extreme capsule consistent with the experimental anatomical studies in the macaque monkey (Petrides and Pandya, 1988, 2002b; Romanski et al., 1999a), demonstrating that the extreme capsule fiber system (ECFS), originally shown in the macaque monkey (Petrides and Pandya, 1988) to link association auditory cortex with the ventrolateral prefrontal region also exists in the human brain. In two subjects, a connection from the Broca ROI to the $\mathrm{ROI}$ in the inferior parietal lobule (44 connection) could not be identified and, in two different subjects, a connection from the Broca ROI to the ROI in the superior temporal gyrus, anterior to Heschl's gyrus, could not be identified.

The group data demonstrate the degree of overlap of the two distinct pathways, i.e., the third branch of the superior longitudinal fasciculus (SLF III) and the ECFS, as well as the individual variability among the different subjects (Fig. 4). 


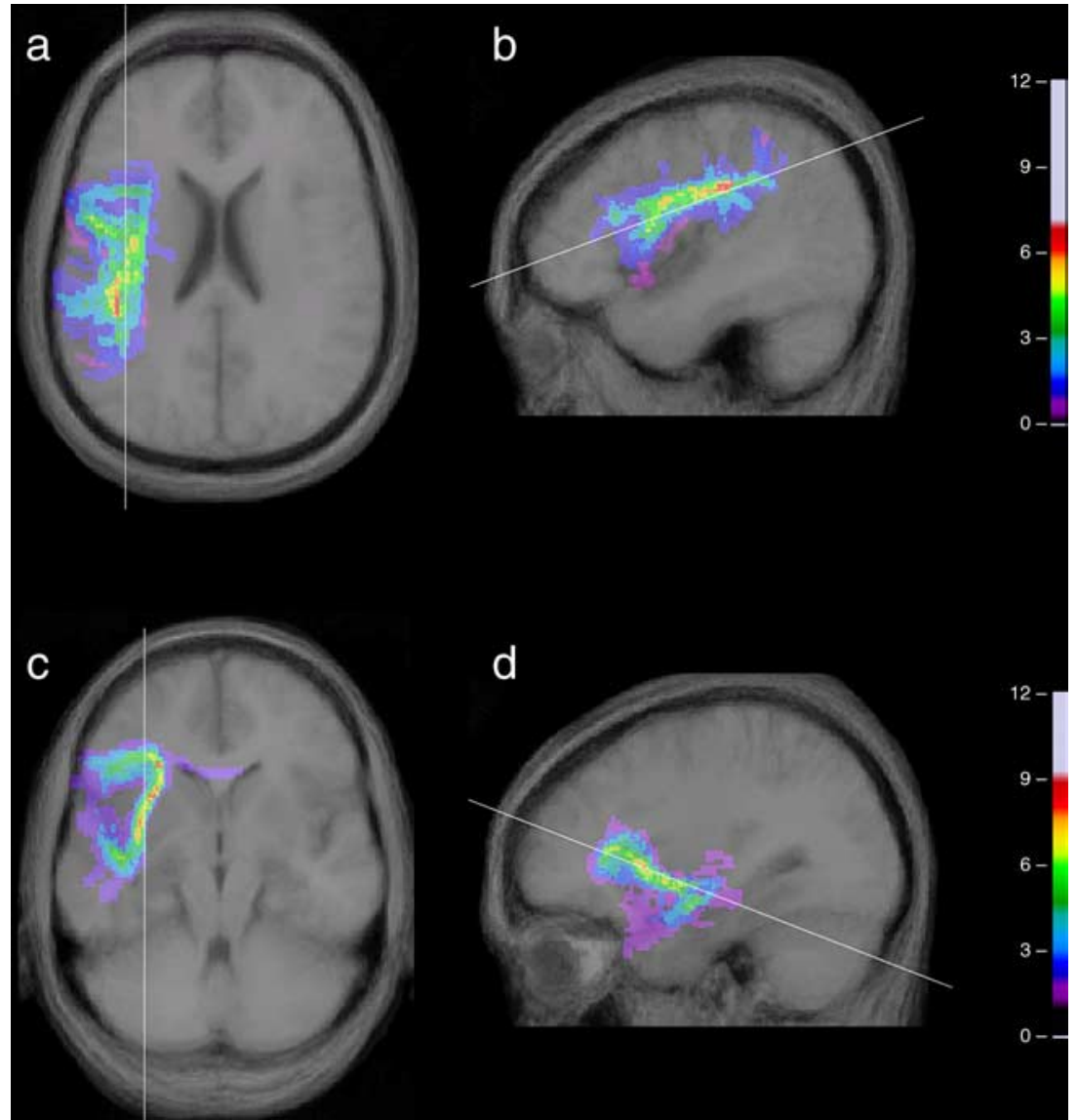

Figure 4. Group data showing connections from areas 44 and 45: MIPs of the sum of the coregistered individual connectivity maps for these connections. The MIP of the group sum connectivity maps for the connection from the ROI in Broca's area to the ROI in the rostral inferior parietal lobule within the supramarginal gyrus $(\boldsymbol{a}, \boldsymbol{b})$, and the connection from the ROI in Broca's area and the $\mathrm{ROI}$ in the superior temporal gyrus and sulcus $(\boldsymbol{c}, \boldsymbol{d})$, depicted in the left hemisphere for the 12 subjects. The former projects from area 44 and the latter from area 45 . The projections are superimposed on the average anatomical brain of the 12 subjects presented here in the axial and sagittal planes. The color intensity scales refer to the amount of overlap for each pathway for all 12 subjects. Brighter colors signify greater overlap.

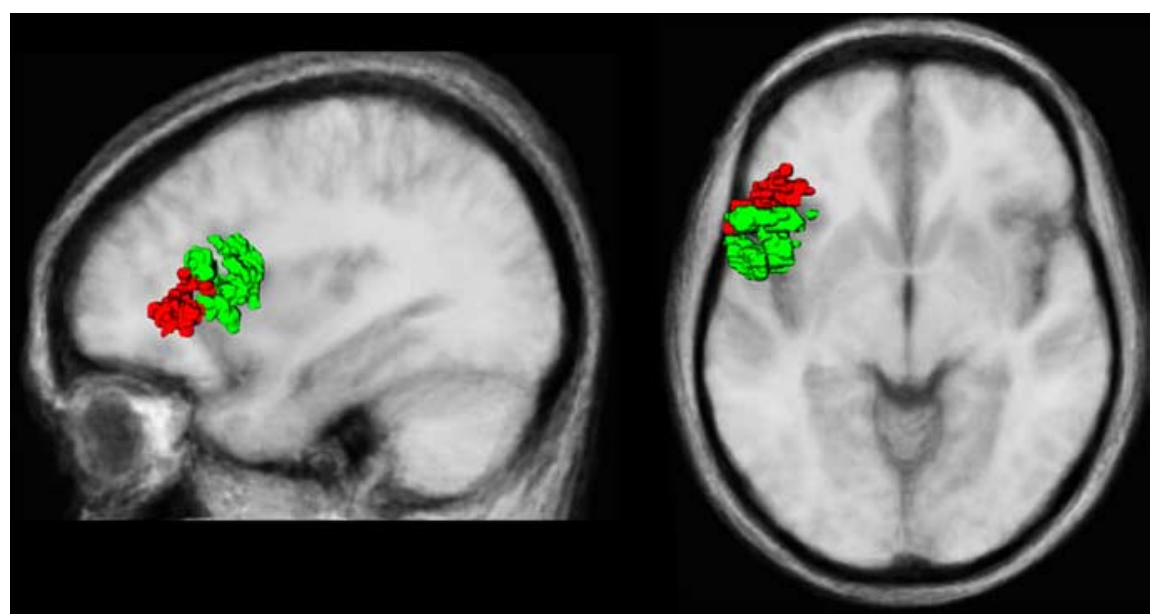

Figure 5. Dissociation of the projections from Broca's area. Group data demonstrating the dissociation of the projections from areas 44 (green) and 45 (red) in all subjects in the left hemisphere. Only the part of the pathway that lay within the ROI centered on Broca's area is shown. The data have been superimposed onto the average anatomical brain of the 12 subjects. An anterior to posterior dissociation of Broca's area is evident.

\section{Dissociation of areas 44 and 45}

It is clear from the Broca's area ROI segmentation results that there is an anterior to posterior division of the connections in this region (Fig. 5). Connections from the anterior extent of Broca's region, which includes the pars triangularis (area 45), course through the ECFS on their way to the superior temporal gyrus and sulcus, whereas fibers from the posterior extent of Broca's region, which includes the pars opercularis (area 44), course through the SLF III on their way to the rostral inferior parietal lobule. These results are entirely consistent with experimental anatomical tract tracing data obtained in the monkey (Petrides and Pandya, 1988, 2002b; Romanski et al., 1999a) and demonstrate the distinct connectivity of the pars opercularis (area 44) versus the pars triangularis (area 45) that together constitute Broca's region.

Additional frontotemporal connections The tractography results for the connectivity between the most posterior part of the superior temporal gyrus and the caudal dorsolateral frontal cortex (area 8 and rostral area 6) revealed connections in 9 of the 12 subjects examined. The fibers followed a course that arched around the caudal extent of the Sylvian fissure toward the ROI in the caudal dorsolateral frontal cortex (Fig. 6, orange tract). These results are in agreement with the experimental anatomical data in the monkey (Petrides and Pandya, 1988, 2002b; Romanski et al., 1999a) demonstrating that fibers from the most posterior part of the superior temporal gyrus arch and enter the white matter of the rostral inferior parietal lobule and continue to caudal dorsolateral frontal cortex.

It should be pointed out here that we also noted the classical arcuate fasciculus pathway (Fig. 6, blue tract) demonstrated in several recent investigations (Catani et al., 2005; Croxson et al., 2005; Parker et al., 2005; Friederici et al., 2006; Powell et al., 2006; Anwander et al., 2007; Rilling et al., 2008). This connection was seen in 12 of 12 subjects, connecting the posterior extent of the superior temporal gyrus to Broca's area. In 7 of 12 subjects, this connection was made to area 44 alone, whereas in the remaining 5 subjects, this connection was made to both areas 44 and 45 but never to area 45 alone.

This projection is similar to that found by other researchers investigating the connections of the human brain (see Discussion). The arching fibers that course around the caudal end of the Sylvian fissure and which form the arcuate pathway have been assumed to link Broca's area with the posterior temporal region and have been observed in both gross dissections of the human brain and modern DTI studies. However, the experimental anatomical autoradiographic 


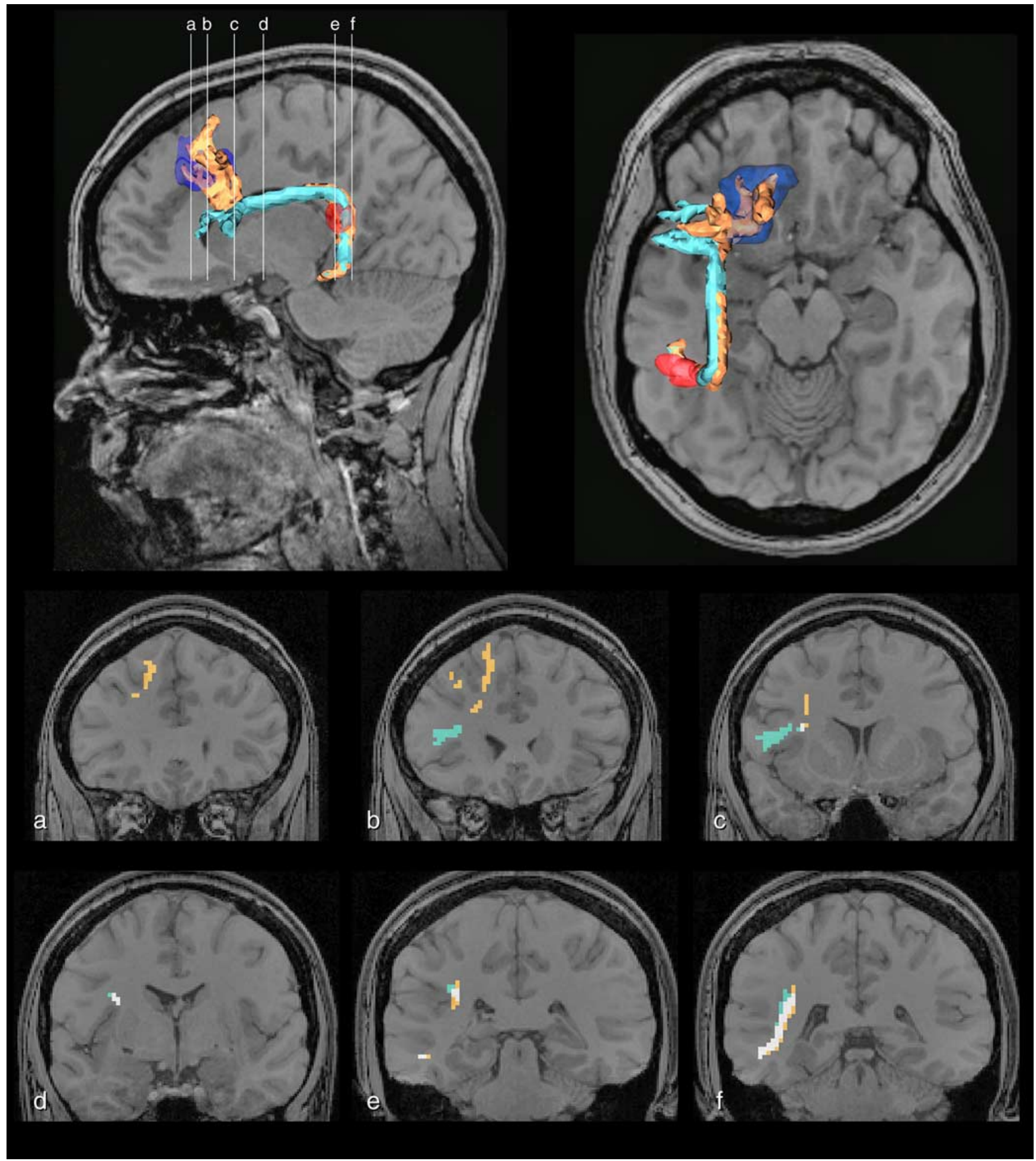

Figure 6. Tractography in a single subject. The tractography results revealed that the ROl at the most posterior extent of the superior temporal gyrus in the left hemisphere (equivalent to the monkey's Tpt region) (red), and the ROl that was located in areas 8 and rostral area 6 (blue) were connected via a pathway that courses around the Sylvian fissure and comes into close proximity with the SLF III (orange tract). The resolution of the voxel size used limits the separation of these individual pathways as evidenced by a connection between this same posterior ROI and the ROI in Broca's area. This is demonstrated in the coronal sections within a single subject $(\boldsymbol{a}-\boldsymbol{f})$. Areas of overlap between the two pathways are shown in white in the coronal sections.

tracing method in the macaque monkey, which allows the precise identification of the origin, trajectory and termination of axons in the brain, have shown that several distinct pathways run in close proximity to each other within the white matter of the rostral inferior parietal lobule. These fiber tracts (SLFII, SLFIII, FOF, and $\mathrm{AF}$ ) would be impossible to separate with current diffusion
MRI methodology, even when using imaging techniques that are robust to the presence of crossing fibers (i.e., the present study) (Parker et al., 2005; Powell et al., 2006; Rilling et al., 2008). Furthermore, with all diffusion imaging studies, the spatial resolution, angular resolution, and signal-to-noise ratio of the data greatly impact the ability to reconstruct corticocortical fiber con- 


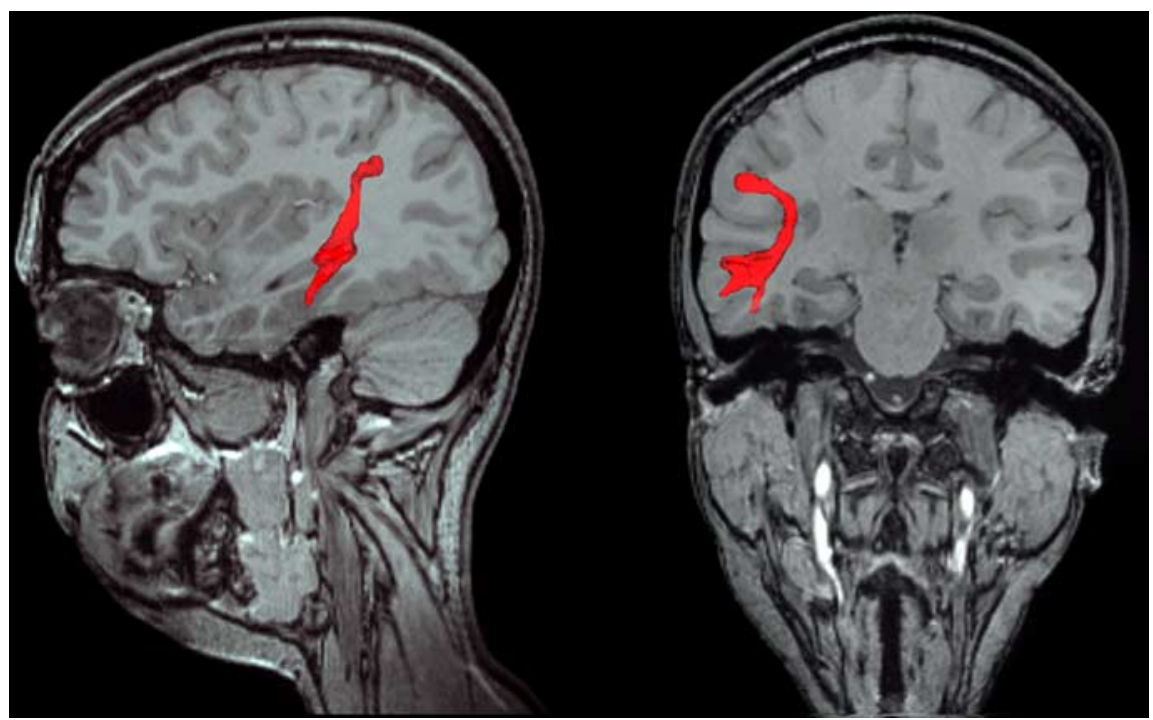

Figure 7. Tractography in a single subject demonstrating connections from the posterior left temporal cortex to the ventral parietal region. The tractography results revealed that there are connections in the human brain that link the caudal superior temporal region to the inferior parietal region. These connections (MDLF; ILF) likely originate to convey auditory and visual information.

nections (see below, Limitations of diffusion-weighted data and Discussion).

\section{Temporal-parietal projections}

The tractography results revealed that, in all 12 subjects, a connection was present between the left ventral posterior intraparietal sulcal region and the ROI in the posterior superior temporal region. These fibers would be running as part of the middle longitudinal fasciculus (MDLF) (Makris et al., 2008) and in the monkey they are also the most caudal part of the inferior longitudinal fasciculus (ILF) (Petrides and Pandya, 2002b, 2006) (Fig. 7). Once again, limitations of the diffusion methodology do not allow us to make a distinction between individual fibers bundles such as the MDLF and the ILF, although we can demonstrate that there are fibers that make direct connections with these two areas (represented as one tract in Fig. 7). These two pathways (MDLF and ILF) are included to demonstrate that information in the vicinity of the caudal Sylvian fissure reaches the inferior parietal lobule and is likely carrying auditory and visual information. These temporoparietal fibers that enter the white matter just below the inferior parietal lobule would likely be incorrectly included with fibers considered to be the arcuate fasciculus in both the classical gross dissections and more recent studies using diffusion-weighted imaging to investigate the language pathways (Catani et al., 2005; Croxson et al., 2005; Parker et al., 2005; Friederici et al., 2006; Powell et al., 2006; Anwander et al., 2007; Rilling et al., 2008), including the present study, because of the difficulty of resolving individual connections given the low resolution of diffusion data.

\section{Limitations of diffusion-weighted data}

Diffusion-weighted MRI data have the same interpretation problems that are encountered when examining the connections of the human brain using the classic gross dissection method: the spatial resolution is limited and therefore corticocortical pathways that run in close proximity to each other cannot be separated. Diffusion MRI is obtained by applying magnetic field gradients in different directions to the subject's head, allowing us to measure a probability distribution function (pdf) for the displacement of water molecules in the tissue at that location. An orientation distribution function (ODF) can then be extracted by integrating along the radial direction of the $3 \mathrm{D}$ pdf to perform further postprocessing of the data and the tracking of pathways in particular. With standard diffusion tensor imaging (DTI), the pdf is modeled using a 3D Gaussian distribution function and, therefore, is limited in situations when voxels present with partial volume effects such as when fibers cross. It is for this reason that we have acquired the data using a model free high angular resolution diffusion imaging (HARDI) approach, which can be used to detect multiple fiber orientations, given by multiple ODF maxima, in individual voxels, unlike DTI techniques (for review, see Campbell et al., 2006; Savadjiev et al., 2006, 2008). The use of high angular resolution makes the detection of fibers that cross other large fiber pathways, such as the SLF III, more likely. However, problems with spatial resolution remain.

One region of the brain that poses particular problems in separating fiber bundles is the white matter of the supramarginal gyrus and the intraparietal region. It is in this white matter location that the AF, the SLF II and III, the MDLF, and the FOF coexist. Diffusion-weighted data from this region, like gross dissections, cannot resolve the specific fiber bundles that mingle with each other, although our tracking approach is able to resolve the orthogonal crossing fibers of the corpus callosum, the cortical-spinal tract, and the SLF, in this brain region. It should be noted here that the fibers of the arcuate fasciculus cannot be separated from those of the superior longitudinal fasciculus in the white matter of the supramarginal gyrus and, therefore, in interpreting the results one should make reference to the macaque monkey experimental anatomical data that can establish the precise origin, course and terminations of fiber bundles. The arcuate connection from the posterior superior temporal gyrus to areas 8 and 6 in the frontal lobe known from macaque monkey studies was observed in 9 of 12 subjects and the classic arcuate projection from the posterior superior temporal gyrus to the ventrolateral frontal region in Broca's area (Fig. 6, blue tract) was seen in 12 of 12 subjects. Of considerable interest is the fact that when we investigated the connections of the MDLF and the ILF (posterior superior temporal ROI to the posterior parietal ROI), all 12 of our subjects demonstrated a projection linking these two areas of the brain together (Fig. 7), and the majority of our subjects also had a connection that continued to area 44 of Broca's area. As noted above, the connections from the most posterior superior temporal gyrus in the monkey lead to areas of the dorsolateral frontal cortex, not to Broca's area, yet because of the proximity of the arcuate fasciculus and the SLF III, these pathways became indistinguishable. Although there is likely a twostage connection (posterior superior temporal region projecting to inferior parietal cortex which, in turn, sends separate fibers to area 44) connecting posterior language areas with the frontal ones, it is by no means the only pathway or even the major one.

It must be noted that all the regions of interest included white matter directly adjacent to the cortex and, therefore, because our 
tractography method, as do others, relies heavily on the anisotropy of diffusion as well as the angular resolution of the diffusion imaging data, fiber directions are harder to extract the closer the tracking is to cortex. This decrease in the coherence of the white matter fiber tracts is attributable to curvature and splay of the fibers, as well as partial volume averaging with gray matter.

Our tractography method is also dependent on the location of the ROIs; only tracts that passed through both ROIs were kept. Therefore anatomical variability from subject to subject is able to influence the tractography results and may explain why in some cases we failed to see a tract (e.g., only 10 of 12 subjects displayed a projection from area 44 to the anterior inferior parietal region). A strong a priori knowledge of the pathways established in experimental anatomical studies in the monkey is key to the correct interpretation of diffusion-weighted data that lack the resolution of the monkey data.

\section{Discussion}

We used diffusion imaging to examine the anatomical pathways connecting the ventrolateral frontal language zone (Broca's region) with posterior cortical regions in the human brain. There was a clear dissociation of the trajectory of fibers from the anterior extent (area 45) of this region compared with those from its posterior extent (area 44) (Figs. 3-5). Area 44 connections are made with the inferior parietal cortex via the third branch of the superior longitudinal fasciculus (SLF III) and area 45 connections are made with the middle section of the superior temporal gyrus, just in front of Heschl's gyrus, via the extreme capsule fiber system (ECFS). These distinct connections of areas 44 and 45 via the SLFIII and the ECFS (Fig. 8) are entirely consistent with the experimental anatomical tracing studies in the macaque monkey which can establish unambiguously the precise origins (i.e., the neurons) and terminations of the axons that form these pathways (Petrides and Pandya, 1984, 1988, 2002b; Romanski et al., 1999a). Thus, although a classical arching pathway (AF) in the human brain may connect posterior language areas with frontal ones, it is by no means the only pathway, as the present results indicate.

In the macaque monkey, an arching pathway links the posterior part of the superior temporal gyrus with the posterior part of the dorsolateral frontal lobe, i.e., area 8 and rostral area 6 (Petrides and Pandya, 1988, 2002b, Romanski et al., 1999a). We examined whether this is also true of the human brain and were able to extract an arcuate fasciculus (AF) coursing from the caudal aspect of the superior temporal gyrus, around the most posterior extent of the Sylvian fissure, and projecting to posterior dorsolateral frontal cortex (area 8 and rostral area 6) in 9 of 12 human brains (Fig. 6, orange fiber tract). These results suggest that, regardless of any additional connections that the arcuate fasciculus may have with ventrolateral frontal cortex in the human brain, it also has connections with posterior dorsolateral frontal cortex and provide a more complete view of the anatomical circuits that link the posterior human language areas.

Area 44, which is connected directly with premotor area 6, and which has recently been shown to be involved with the orofacial musculature (Petrides et al., 2005) is likely responsible for the higher-order articulatory control of speech. Area 45, on the other hand, which has direct connections with area 44 and with the auditory belt area of the superior temporal region via the extreme capsule, may be more involved in the top-down regulation of linguistic processing involved in verbal retrieval (see Petrides, 2006). More specifically, area 45 , which has been shown to be involved in the active retrieval of information from nonverbal memory in monkeys (Cadoret and Petrides, 2007), nonverbal and verbal memory in humans (Petrides et al., 1995; Petrides, 2002; Amunts et al., 2004; Kostopoulos and Petrides, 2008), and verbal fluency (Amunts et al., 2004) is in an ideal position to retrieve certain aspects of verbal information that aid in language communication in the left hemisphere of the human brain.

If the AF is not the only one or even the main pathway implicated in the speech act, what route does auditory information take to the frontal lobe language zone? Electrophysiology and tracer injection studies in the monkey have demonstrated that two separate auditory pathways from the frontal cortex exist (Petrides and Pandya, 1988, 2002b; Romanski et al., 1999a,b). It has been shown that different clusters of neurons within the auditory belt region of the superior temporal gyrus respond selectively to auditory stimuli (Rauschecker and Tian, 2000; Tian et al., 2001). For instance, the lateral auditory belt, which is connected to ventrolateral frontal area 45, responds to the type of calls that an animal makes, whereas the more posterior extent of the auditory belt, which is connected to the caudal dorsolateral frontal areas (i.e., area 8 and rostral area 6), is involved in spatially selective auditory stimuli (Leinonen et al., 1980). These findings have led to the proposal that a more anterior section of the auditory belt region may be involved in an anterior auditory object recognition stream and the posterior section in an auditory spatial stream (Rauschecker and Tian, 2000). Human neuroimaging studies have corroborated the animal data demonstrating a func- 
tional involvement of the more anterior auditory belt region in vocal processing (Belin et al., 2000; Binder et al., 2000; Scott et al., 2000; Obleser et al., 2006). In contrast, it has been suggested that the posterior auditory belt region may be involved in sound localization and processing of sound motion in space (Warren et al., 2002; Zatorre et al., 2002; Krumbholz et al., 2005).

Electrophysiological recording studies have demonstrated that neurons in the intraparietal and rostral inferior parietal cortex of the monkey (the same region where we placed a ROI) are specialized for somatosensory and spatial responses that involve the hand, arm and mouth area (Hyvärinen and Shelepin, 1979; Leinonen and Nyman, 1979). The diffusion-weighted data, in agreement with the monkey literature, has identified the SLF III pathway linking area 44 with the inferior parietal lobule in the majority of the subjects (Figs. 3, 4). Interestingly, another diffusion imaging study in the human brain by Rushworth and colleagues (2006) has also noted the SLF III pathway extending into the ventral premotor and pars opercularis region of the frontal lobe. Neuroimaging data in humans lend additional support to this corticocortical projection, suggesting that the inferior parietal lobule may be responsible for processing more than spatial information, including auditory information involved in both motor and nonmotor tasks (Rauschecker and Tian, 2000) such as speech (Wise et al., 2001).

Additional tracts that need mentioning are the MDLF and the ILF that connect anterior and posterior regions of the inferior parietal cortex with auditory areas in the superior temporal gyrus (Makris et al., 2008) and extrastriate areas of the visual system (Seltzer and Pandya, 1984). These fiber bundles course in a ventral-to-dorsal direction and mingle with the fibers of the SLF II and FOF tracts that connect with areas 8 and 6 in the frontal cortex (Petrides and Pandya, 2006). In the present study, all of the subjects displayed a connection from the extrastriate region of the brain to an ROI that was located in the region of the posterior intraparietal gyrus (Fig. 7).

The focus of earlier studies using DTI to examine the connections of the language zones of the human brain was on the classical arcuate fasciculus connection that was assumed to be the main one (Catani et al., 2005). An alternative route connecting superior temporal gyrus with Broca's region had also been suggested by some earlier studies. Parker et al. (2005) reported in 4 of the 11 subjects examined a "ventral" pathway and suggested that this demonstration may have been at the limit of their tract tracing method. They were also uncertain whether this "ventral" pathway runs through the uncinate fasciculus or the external capsule. Although current DTI methodology does not have the resolution to resolve the external from the extreme capsules that are located so near to each other (one just medial and the other just lateral to the claustrum), the monkey experimental anatomical tracer studies demonstrate that this connection is made via the extreme capsule (Petrides and Pandya, 2002b). Croxson et al. (2005) and Anwander et al. (2007) also suggested the existence of this "ventral" pathway. The present study has provided strong evidence for the existence of this pathway that is strongly predicted from the monkey work.

In conclusion, consistent with experimental anatomical work in the macaque monkey, two divergent and direct pathways emerge from the two architectonically distinct components of Broca's region: from area 44, the SLF III to the intraparietal and rostral inferior parietal region and from area 45, the ECFS to the temporal lobe. It should be noted that there are strong connections between the superior temporal region and the inferior parietal region and given the current resolution of DTI some of these temporoparietal fibers are likely to be included in the demonstrated arcuate fasciculus. Thus, it remains an open question whether new direct connections have developed in the human brain between posterior temporal cortex and Broca's region via the arcuate fasciculus or whether the posterior temporal cortex projects to inferior parietal cortex which in turn projects to area 44 and this distinction cannot be resolved with current DTI methodology. Also, limitations associated with diffusion data may be a reason why the AF is not seen clearly in the monkey (see Results, Limitations of diffusion-weighted data).

Language in the human likely developed, not only because Broca's region increased in size, but from an overall cortical expansion that necessarily included larger fiber tracts throughout the brain and which can be viewed as conflated in some regions when processing diffusion data. As diffusion imaging and processing techniques improve, so too will our understanding of the connectivity underlying the human brain.

\section{References}

Amunts K, Schleicher A, Bürgel U, Mohlberg H, Uylings HB, Zilles K (1999) Broca's region revisited: cytoarchitecture and intersubject variability. J Comp Neurol 412:319-341.

Amunts K, Weiss PH, Mohlberg H, Pieperhoff P, Eickhoff S, Gurd JM, Marshall JC, Shah NJ, Fink GR, Zilles K (2004) Analysis of neural mechanisms underlying verbal fluency in cytoarchitectonically defined stereotaxic space-the roles of Brodmann areas 44 and 45 . Neuroimage 22:42-56.

Anwander A, Tittgemeyer M, von Cramon DY, Friederici AD, Knösche TR (2007) Connectivity-based parcellation of Broca's area. Cereb Cortex $17: 816-825$.

Belin P, Zatorre RJ, Lafaille P, Ahad P, Pike B (2000) Voice-selective areas in human auditory cortex. Nature 403:309-312.

Binder JR, Frost JA, Hammeke TA, Bellgowan PSF, Springer JA, Kaufman JN, Possing ET (2000) Human temporal lobe activation by speech and nonspeech sounds. Cereb Cortex 10:512-528.

Cadoret G, Petrides M (2007) Ventrolateral prefrontal neuronal activity related to active controlled memory retrieval in nonhuman primates. Cereb Cortex 17:i27-i40.

Campbell JSW, Savadjiev P, Siddiqi K, Pike GB (2006) Validation regularization in diffusion MRI tractography. In: Third IEEE international symposium on biomedical imaging: nano to macro, 2006, pp. 351-354.

Catani M, Jones DK, ffytche DH (2005) Perisylvian language networks of the human brain. Ann Neurol 57:8-16.

Conturo TE, Lori NF, Cull TS, Akbudak E, Snyder AZ, Shimony JS, McKinstry RC, Burton H, Raichle ME (1999) Tracking neuronal fiber pathways in the living human brain. Proc Natl Acad Sci USA 96:10422-10427.

Croxson PL, Johansen-Berg H, Behrens TEJ, Robson MD, Pinsk MA, Gross CG, Richter W, Richter MC, Kastner S, Rushworth MFS (2005) Quantitative investigation of connections of the prefrontal cortex in the human and macaque using probabilistic diffusion tractography. J Neurosci 25:8854-8866.

Friederici AD, Bahlmann J, Heim S, Schubotz RI, Anwander A (2006) The brain differentiates human and non-human grammars: functional localization and structural connectivity. Proc Natl Acad Sci USA 103:2458-2463.

Geschwind N (1970) The organization of language in the brain. Science 170:940-944.

Grodzinsky Y, Amunts K (2006) Broca's region. New York: Oxford UP.

Hyvärinen J, Shelepin Y (1979) Distribution of visual and somatic functions in the parietal associative area 7 of the monkey. Brain Res 169:561-564.

Kostopoulos P, Petrides M (2008) Left mid-ventrolateral prefrontal cortex: underlying principles of function. Eur J Neurosci 27:1037-1049.

Krumbholz K, Schönwiesner M, von Cramon DY, Rübsamen R, Shah NJ, Zilles K, Fink GR (2005) Representation of interaural temporal information from left and right auditory space in the human planum temporale and inferior parietal lobe. Cereb Cortex 15:317-324.

Leinonen L, Nyman GII (1979) Functional properties of cells in anterolateral part of area 7 associative face area of awake monkeys. Exp Brain Res 34:321-333. 
Leinonen L, Hyvärinen J, Sovijärvi ARA (1980) Functional properties of neurons in the temporo-parietal association cortex of awake monkey. Exp Brain Res 39:203-215.

MacDonald D (1996) MNI-display: program for display and segmentation of surfaces and volumes. Retrieved August 21, 2008, from http://www.bic.mni.mcgill.ca/software/Display/Display.html.

Maes F, Collignon A, Vandermeulen D, Marchal G, Suetens P (1997) Multimodality image registration by maximization of mutual information. IEEE Trans Med Imaging 16:187-198.

Makris N, Papadimitriou GM, Kaiser JR, Sorg S, Kennedy DN, Pandya DN (2008) Delineation of the middle longitudinal fascicle in humans: a quantitative, in vivo, DT-MRI study. Cereb Cortex. Advance online publication. Retrieved October 10, 2008. doi:10.1093/cercor/bhn124.

Mori S, Crain BJ, Chacko VP, van Zijl PCM (1999) Three dimensional tracking of axonal projections in the brain by magnetic resonance imaging. Ann Neurol 45:265-269.

Obleser J, Boecker H, Drzezga A, Haslinger B, Hennenlotter A, Roettinger M, Eulitz C, Rauschecker JP (2006) Vowel sound extraction in anterior superior temporal cortex. Hum Brain Mapp 27:562-571.

Ojemann G, Ojemann J, Lettich E, Berger M (1989) Cortical language localization in left, dominant hemisphere. J Neurosurg 71:316-326.

Parker GJM, Luzzi S, Alexander DC, Wheeler-Kingshott CAM, Ciccarelli O, Lambon Ralph MA (2005) Lateralization of ventral and dorsal auditorylanguage pathways in the human brain. Neuroimage 24:656-666.

Penfield W, Roberts L (1966) Speech and brain-mechanisms. New York: Atheneum.

Petrides M (2002) The mid-ventrolateral prefrontal cortex and active mnemonic retrieval. Neurobiol Learn Mem 78:528-538.

Petrides M (2006) Broca's area in the human and the non-human primate brain. In: Broca's region (Amunts K, Grodzinsky Y, eds), pp 31-46. Oxford: Oxford UP.

Petrides M, Pandya DN (1984) Projections to the frontal cortex from the posterior parietal region in the rhesus monkey. J Comp Neurol 228:105-116.

Petrides M, Pandya DN (1988) Association fiber pathways to the frontal cortex from the superior temporal region in the rhesus monkey. J Comp Neurol 273:52-66.

Petrides M, Pandya DN (1994) Comparative cytoarchitectonic analysis of the human and the macaque frontal cortex. In: Handbook of neuropsychology (Boller F, Grafman J, eds), Vol 9, pp 17-58. Amsterdam: Elsevier Science.

Petrides M, Pandya DN (2002a) Comparative cytoarchitectonic analysis of the human and the macaque ventrolateral prefrontal cortex and corticocortical connection patterns in the monkey. Eur J Neurosci 16:291-310.

Petrides M, Pandya DN (2002b) Association pathways of the prefrontal cortex and functional observations. In: Principles of frontal lobe function (Stuss DT, Knight RT, eds), pp 31-50. New York: Oxford UP.

Petrides M, Pandya DN (2006) Efferent association pathways originating in the caudal prefrontal cortex in the macaque monkey. J Comp Neurol 498:227-251.
Petrides M, Alivisatos B, Evans AC (1995) Functional activation of the human ventrolateral frontal cortex during mnemonic retrieval of verbal information. Proc Natl Acad Sci U S A 92: 5803-5807.

Petrides M, Cadoret G, Mackey S (2005) Orofacial somatomotor responses in the macaque monkey homologue of Broca's area. Nature 435:1235-1238.

Powell HWR, Parker GJM, Alexander DC, Symms MR, Boulby PA, WheelerKingshott CAM, Barker GJ, Noppeney U, Koepp MJ, Duncan JS (2006) Hemispheric asymmetries in language-related pathways: a combined functional MRI and tractography study. Neuroimage 32:388-399.

Rasmussen T, Milner B (1975) Clinical and surgical studies of the cerebral speech areas in man. In: Cerebral localization (Zulch, KJ, Creutzfeldt O, Galbraith GC, eds), pp 238-257. Berlin: Springer.

Rauschecker JP, Tian B (2000) Mechanisms and streams for processing of "what" and "where" in auditory cortex. Proc Natl Acad Sci U S A 97:11800-11806.

Rilling JK, Glasser MF, Preuss TM, Ma X, Zhao T, Hu X, Behrens TEJ (2008) The evolution of the arcuate fasciculus revealed with comparative DTI. Nat Neurosci 11:426-428.

Romanski LM, Tian B, Fritz J, Mishkin M, Goldman-Rakic PS, Rauschecker JP (1999a) Dual streams of auditory afferents target multiple domains in the primate prefrontal cortex. Nat Neurosci 2:1131-1136.

Romanski LM, Bates JF, Goldman-Rakic PS (1999b) Auditory belt and parabelt projections to the prefrontal cortex in the rhesus monkey. J Comp Neurol 403:141-157.

Rushworth MFS, Behrens TEJ, Johansen-Berg H (2006) Connection patterns distinguish 3 regions of human parietal cortex. Cereb Cortex 16:1418-1430.

Savadjiev P, Campbell JS, Pike GB, Siddiqi K (2006) 3D curve inference for diffusion MRI regularization and fibre tractography. Med Image Anal 10:799-813.

Savadjiev P, Campbell JS, Descoteaux M, Deriche R, Pike GB, Siddiqi K (2008) Labelling of ambiguous subvoxel fibre bundle configurations in high angular resolution diffusion MRI. Neuroimage 41:58-68.

Scott SK, Blank CC, Rosen S, Wise RJS (2000) Identification of a pathway for intelligible speech in the left temporal lobe. Brain 123:2400-2406.

Seltzer B, Pandya DN (1984) Further observations on parieto-temporal connections in the rhesus monkey. Exp Brain Res 55:301-312.

Tian B, Reser D, Durham A, Kustov A, Rauschecker JP (2001) Functional specialization in rhesus monkey auditory cortex. Science 292:290-293.

Tuch DS, Reese TG, Wiegell MR, Wedeen VJ (2003) Diffusion MRI of complex neural architecture. Neuron 40:885-895.

Warren JD, Zielinski BA, Green GGR, Rauschecker JP, Griffiths TD (2002) Perception of sound-source motion by the human brain. Neuron 34:139-148.

Wise RJS, Scott SK, Blank SC, Mummery CJ, Murphy K, Warburton EA (2001) Separate neural subsystems within 'Wernicke's area'. Brain 124:83-95.

Zatorre RJ, Bouffard M, Ahad P, Belin P (2002) Where is 'where' in the human auditory cortex? Nat Neurosci 5:905-909. 